\title{
3D Printing of a Mandibular Bone Deffect
}

\author{
ALIN GABOR ${ }^{1}$, TIBERIU HOSSZU2*, CRISTIAN ZAHARIA ${ }^{1}$, ALEXANDRU KOZMA ${ }^{1}$, ANDREEA CODRUTA COJ OCARIU1, \\ MEDA LAVINIA NEGRUTIU ${ }^{1}$, CAMELIA SZUHANEK ${ }^{7}$, COSMIN SINESCU ${ }^{1}$ \\ ${ }^{1}$ Victor Babes University of Medicine and Pharmacy of Timisoara, 2 Piata Eftimie Murgu, 300041, Timisoara, Romania \\ 2"Vasile Goldis Western University of Arad, 94 Revolutiei Blvd., 310025, Arad, Romania
}

The aim of this study was to achieve a polymeric scaffold, ex-vivo, using 3D printing technology and then subjecting it to various tests to check its optimal property. Initially there was selected a lower jaw with a bone defect that would have prevented any treatment based prosthetic implant. The mandible was first scanned using an optical scanner (MAESTRO DENTAL SCANNER MDS400). The scanning parameters using optical scanning system are: 10 micron accuracy, resolution $0.07 \mathrm{~mm}, 2$ rooms with High-Resolution LED structured light, two axes. The scan time of the mandible was 4-5 min. Later the same mandible was scanned using CBCT's CRANEX 3DX. The images obtained using CBCT's were correlated with those obtained by optical scanning. Further on, there was achieved the digital design of the future scaffold with the conventional technique of wax addition directly on the mandibular bone defect. After that, this was again scanned using scanning system MAESTRO DENTAL SCANNER MDS400, and using CBCT's CRANEX 3DX. The images obtained were correlated with all the scanned images of original mandible bone defects. There were made two polymeric scaffolds using 3D printing system an (D20 Digital Wax System 3D Printer). After printing, scaffold sites were introduced for 30 minutes in an oven curing. Later the pieces obtained were processed to remove small excesses of work. There were obtained 3 blocks of polymers that have a good adaptation to the bone profile. Often, in oral implantology and maxillofacial surgery appear bone defects. They prevent an optimal treatment of bio-functional and aesthetic restoration. Using 3D printing technology one can achieve scaffold sites of different biocompatible materials that have optimal properties to replace bone defect and restore the defective area. These scaffold sites have an intimate adaptation to the defect. 3D printing techniques used to restore bone defects can quickly and efficiently give the possibility to have a successful implantology prosthetics treatment.

Keywords: polymeric scaffold, 3D printing technology, prosthetic implant, bone defects

Three-dimensional (3D) printing, also known as additive manufacturing $(\mathrm{AM})$, relates to various manufacturing processes used to obtain a 3D object. In 3D printing, successive layers of material are deposited under computer control to create a certain object which can be of almost any shape or geometry [1]. The manufacturing is made using a 3D model or based on other sources of electronic data. The original meaning of the term 3D Printing refers to the process by accumulating sequentially, layer by layer, the material on a bed of powder print heads similar to inkjet [2-4]. More recently, its meaning has expanded to encompass a wider variety of techniques such as extrusion and sintering based processes. 3D printing process requires no molds because of the manufacturing method in which material is added in layers [5-9].

3D printing is also considered distinct from traditional manufacturing techniques, which rely mainly on removing materials by methods such as cutting or drilling (subtractive processes).The term 3D printing, covers a range of processes and technologies that offer a full spectrum of possibilities for the production of parts and products made of different materials. 3D printing applications are still developing [9-15].

\section{Experimental part}

\section{Materials and methods}

Often, in oral implantology and maxillofacial surgery appear bone defects which prevent an optimal treatment of bio-functional and aesthetic restoration. Thus using 3D printing technology can achieve scaffold sites of different biocompatible materials that have optimal properties to replace bone defect and restore defective area. These scaffold sites have an intimate adaptation to the defect. In addition to 3D printing systems, to achieve this scaffold is needed the transposition of the clinical situation or the defect in the system of 3D printing. This is achievable using digital scanning systems, as well as radiographic examination using CBCT. Also scan after achieving clinical situation needs to perform computerized design to achieve 3D printing.

The aim of this study was to achieve a polymeric scaffold, ex-vivo, using 3D printing technology and then subjecting it to various tests to check its optimal properties.

Initially there was selected a mandible with a bone defect that would make impossible any treatment based on a prosthetic implant. Figure 1 shows the mandible with bone defect in quadrant 3 .

The mandible was first scanned using an optical scanner (MAESTRO DENTAL SCANNER MDS400). The scanning parameters using optical scanning system are: 10 micron accuracy, resolution $0.07 \mathrm{~mm}, 2$ rooms with HighResolution LED structured light, two axes. The scan time

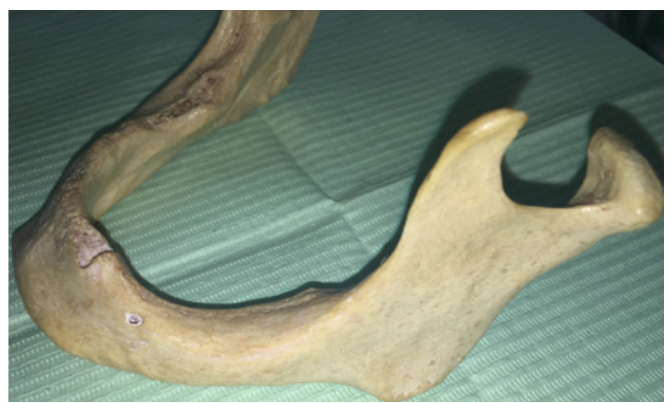

Fig.1.The mandible with bone defect in quadrant 3

\footnotetext{
*Phone: (+40) 0257259853
} 


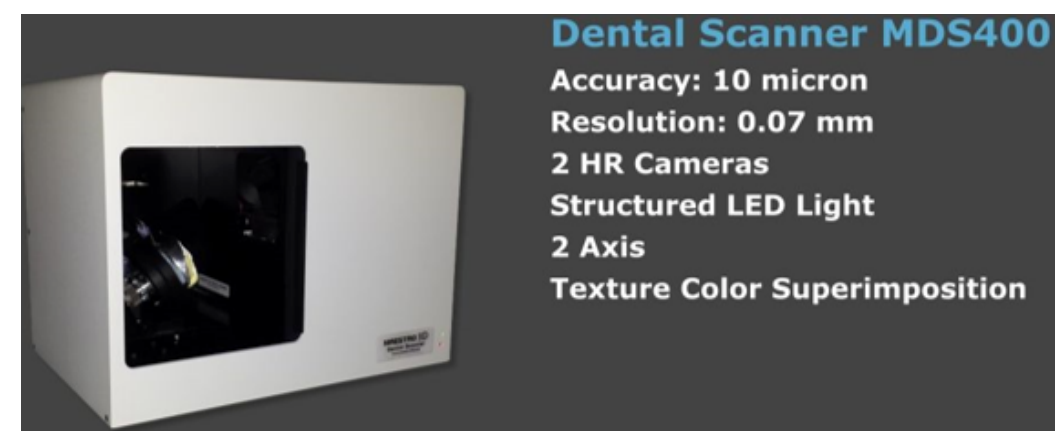

Fig.2. The optical scanner (MAESTRO DENTAL SCANNER MDS400) and the scanning parameters

of the mandible was 4-5 min. Figure 2 shows the optical scanner (MAESTRO DENTAL SCANNER MDS400) and the scanning parameters.

Later the same mandible was scanned using CBCT's CRANEX 3DX. In figure 3 is presented the CBCT.

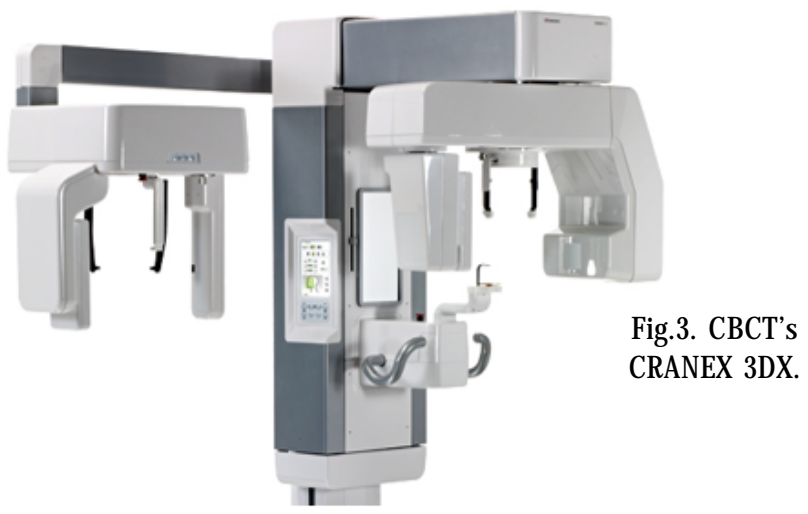

The images obtained using the $\mathrm{CBCT}^{\prime}$ were correlated with those obtained by optical scanning. It was obtained the exact position of the bone defect of the jaw.

Further on, was achieved the digital design of the future scaffold with the conventional technique of wax addition directly on the mandibular bone defect. Figure 4 shows the wax design of the future scaffold.

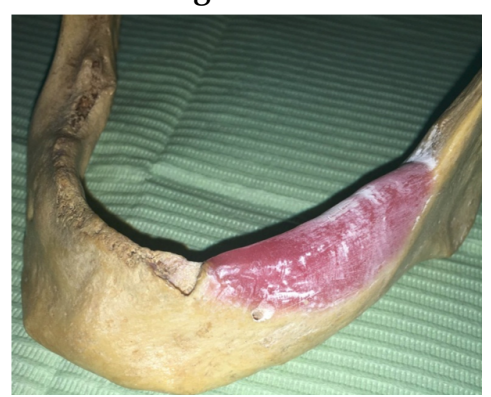

Fig.4. The wax design of the future scaffold.

In figure 4 we can also see the powder that was used for 3D scanning system. The powder used is named Okklean Occlusion Spray and is needed in the protocol of 3D scanning to prevent the light reflection of the surface examined, and so, to prevent blind spots on the 3D image reconstructed on the monitor.

The mandible was again scanned using scanning system MAESTRO DENTAL SCANNER MDS400, and using CBCT'S CRANEX 3DX. The images obtained were correlated with all the scanned images of original mandible bone defects. A two digital design of the future scaffold was obtained: (i) one by optical scanning of the wax design and correlated with the data of the jaw bone defect scanned initially and (ii) one by scanning using CBCT of the wax design. In consequence, two 3D printed scaffolds were made from polymeric material using the Digital 3D Printer Wax System D2. Figure 5 and 6 show the 3D printed scaffolds made from polymeric material, the unprocessed one and the final one. The resin that the 3D Printer uses for

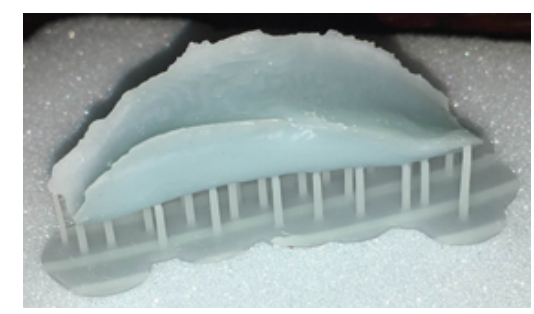

Fig.5. The 3D printed unprocessed scaffold made from polymeric material

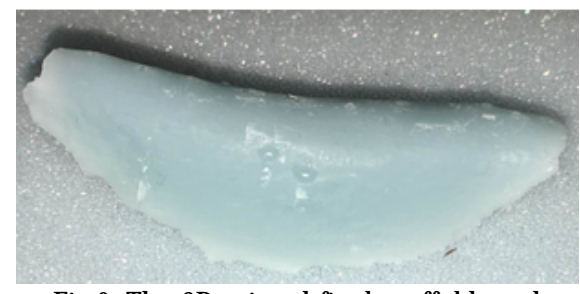

Fig.6. The 3D printed final scaffold made from polymeric material.

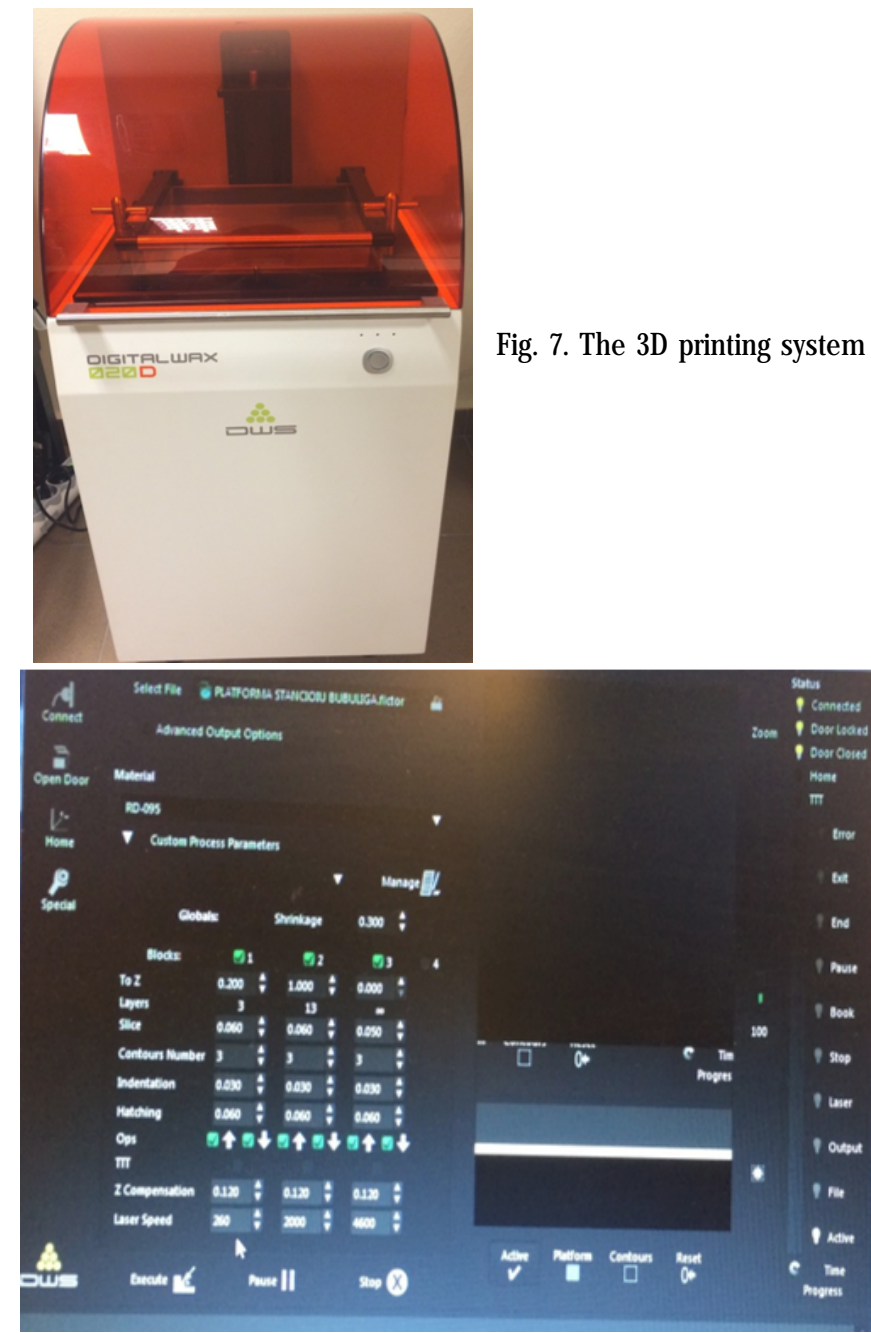

Fig.8. The 3D printing system parameters

printing is a photosensitive resin named DigitalWax $₫$ DCSeries Casting Resin.

So there were made two polymeric scaffolds using 3D printing system (D20 Digital Wax System 3D Printer). Figure 7 shows the $3 D$ printing system. This is a 3D printing system that prints light curing resins using laser, which are laminated.

3D printing system parameters are as follows: printing can be carried out in slices (slice) of a thickness ranging from $0.01-0.10 \mathrm{~mm}$ at a temperature of $22-25^{\circ} \mathrm{C}$ and a relative humidity of $60 \%$. The $3 \mathrm{D}$ printing time of a scaffold 


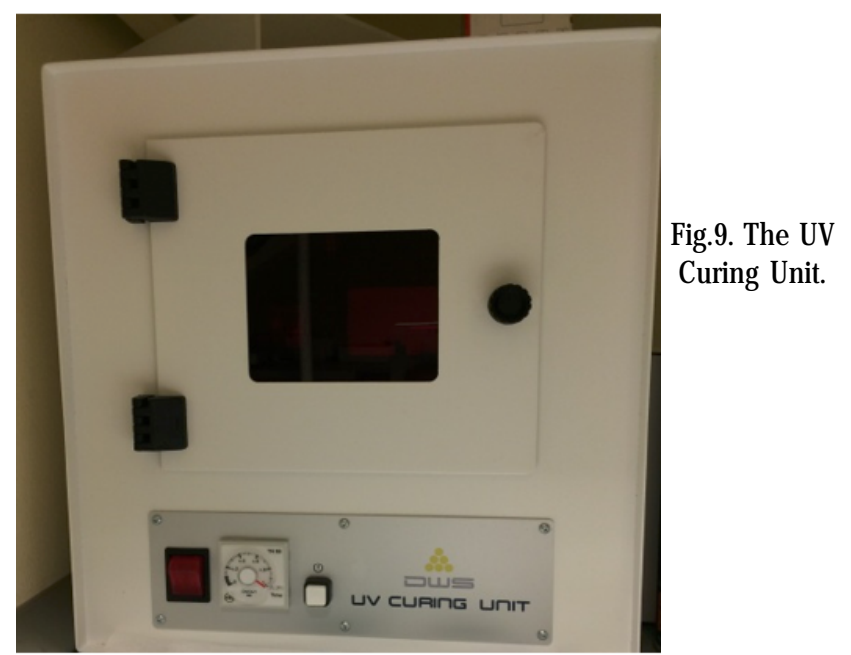

was about $\pm 4 \mathrm{~h}$. Figure 8 shows the 3D printing system parameters.

After printing, scaffold sites were introduced for 30 minutes in an oven curing. Later the pieces obtained were processed to remove small excesses of work. Figure 8 shows the curing oven wich is from same 3D printing system.

\section{Results and discussions}

The scaffolds of polymers resulted after being followed all the steps of 3D scanning and 3D printing had a good adaptation to the bone profile. However, capturing the image of bone defect in vivo is better to be realised using CBCT, with the 3D scanning being necessarily to develop the bone in order to see the defect. Using radiographic examination is gained time to better manage and analyse the clinical situation. In this case, was a in vitro study, so there were no differences to be mentioned between the $3 \mathrm{D}$ printing and $\mathrm{CBCT}$.

Bone defects are found often in oral implantology and maxillofacial surgery [16-18]. An optimal treatment means to create a restoration that is both bio-functional and aesthetic. Using 3D printing technology there can be achieved scaffold sites of different biocompatible materials that have optimal properties to replace bone defect and restore the defective area. These scaffold sites have an intimate adaptation to the defect. The human error is absent of the equation, because the computer calculates and creates the final product, which in this case was the reconstructed scaffold [19-21].

This was an in vitro study. In an in vivo study appear more factors to take into consideration, which can lead to different errors in capturing the data. The scanning process can be achieved just after developing the bone defect, and a $4 \mathrm{~h}$ waiting for the 3D printing is impossible, the time needed for surgery would be too much. Instead, using CBCT, this issue is solved, because the image of the bone defect is radiographic captured, so non-invasive, the team can prepare the clinical case and avoid all the errors that can appear during the surgery of bone reconstruction. Also, by well knowing and understanding the protocol of capturing the 3D image of the bone defect, can be avoided the all the errors and the blind spots on the 3D reconstructed area.

\section{Conclusions}

3D printing techniques used to restore bone defects can quickly and efficiently give the possibility to have a successful implantology prosthetics treatment.

In order to obtain the best results is necessary to prevent the errors that can occur during capturing the data needed in order to print the scaffold.

\section{References}

1.3D Printer Technology - Animation of layering. Create It Real. Retrieved 2012-01-31.

2. DOICIN, C.V., ULMEANU, M.E., NEAGU, C., POPA, L., Mat. Plast., 53, no. 1,2016, p. 139

3. PASCU, N., ARION, A.F., DOBRESCU, T., CARUTASU, N.L., Mat. Plast., 52, no. 2, 2015, p. 141

4. BURDE, A.V., DUDEA, D., CUC, S., MOLDOVAN, M., CAMPIAN, R.S., Mat. Plast., 53, no. 1, 2015, p. 65

5.J EREMY RIFKIN and The Third Industrial Revolution Home Page. Thethirdindustrialrevolution.com. Retrieved 2016-01-04.

6.***A third industrial revolution. The Economist. 2012-04-21. Retrieved 2016-01-04

7.J ANE BIRD (2012-08-08). Exploring the 3D printing opportunity. The Financial Times. Retrieved 2012-08-30.

8.HIDEO KODAMA, A Scheme for Three-Dimensional Display by Automatic Fabrication of Three-Dimensional Model, IEICE TRANSACTIONS on Electronics (Japanese Edition), vol.J64-C, No.4, pp.237-241, April 1981

9.HIDEO KODAMA, Automatic method for fabricating a threedimensional plastic model with photo-hardening polymer, Review of Scientific Instruments, Vol. 52, No. 11, pp. 1770-1773, November 1981 10.JEAN-CLAUDE, ANDRE. Disdpositif pour realiser un modele de piece industrielle.National De La Propriete Industrielle.

11.MENDOZA, HANNAH ROSE (May 15, 2015). Alain Le Mehaute, The Man Who Submitted Patent For SLA 3D Printing Before Chuck Hull. 3dprint.com.

12.MOUSSION, ALEXANDRE (2014). Interview d'Alain Le Méhauté, l'un des pères de l'impression 3D". Primante 3D.

13.***3D Printing: What You Need to Know. PCMag.com. Retrieved 2013-10-30.

14.***Apparatus for Production of Three-Dimensional Objects by Stereolithography (8 August 1984)

15. FREEDMAN, DAVID H. Layer By Layer. Technology Review 115.1 (2012): 50-53. Academic Search Premier. Web. 26) uly 2013.

16. RUSU, L.C., PIRTE, A., BORTUN, C.M., HAINOIU, B., PODARIU, A.C., ARDELEAN, L., NEGRUTIU, M.L., SINESCU, C., PLES, H., Rev. Chim. (Bucharest), 66, no.2, 2015, p. 223

17. RUSU, L.C., SECHE, E., FREIMAN, P.C., HAINOIU, B., NEGRUTIU, M.L., ARDELEAN, L., SINESCU, C., Rev. Chim. (Bucharest), 65, no. 9, 2014, p. 1114

18. RUSU, L.C., PIRTE, A., BORTUN, C.M., HAINOIU, B., PODARIU, A.C., ARDELEAN, L., NEGRUTIU, M.L., SINESCU, C., PLES, H., Rev. Chim. (Bucharest), 66, no. 3, 2015, p. 396

19.AMON, C. H.; BEUTH, J. L.; WEISS, L. E.; MERZ, R.; PRINZ, F. B. (1998). Shape Deposition Manufacturing With Microcasting: Processing, Thermal and Mechanical Issues (PDF).J ournal of Manufacturing Science and Engineering 120 (3). Retrieved 2014-12-20.

20.BECK, J.E.; FRITZ, B.; SIEWIOREK, DANIEL; WEISS, LEE (1992). Manufacturing Mechatronics Using Thermal Spray Shape Deposition (PDF). Proceedings of the 1992 Solid Freeform Fabrication Symposium. Retrieved 2014-12-20.

21.PRINZ, F. B.; MERZ, R.; WEISS, LEE (1997). IKAWA, N., ed. Building Parts You Could Not Build Before. Proceedings of the 8th International Conference on Production Engineering. 2-6 Boundary Row, London SE1 8HN, UK: Chapman \& Hall. pp.40-44

Manuscript received: 3.02.2017 